03

\title{
Неэкспоненциально затухающие электромагнитные волны, локализованные на границе анизотропных металлодиэлектрических структур
}

\author{
(C) Н.С. Петров ${ }^{1}$, С.Н. Курилкина ${ }^{2}$, А.Б. Зимин ${ }^{3}$, В.Н. Белый ${ }^{2}$ \\ ${ }^{1}$ Институт повышения квалификации по новым направлениям развития техники, технологий и экономики \\ Белорусского национального технического университета, \\ 220107 Минск, Беларусь \\ ${ }^{2}$ Институт фризики им. Б.И. Степанова НАН Беларуси, \\ 220072 Минск, Беларусь \\ ${ }^{3}$ Белорусский государственный университет информатики и радиоэлектроники, \\ 220013 Минск, Беларусь \\ e-mail: s.kurilkina@ifanbel.bas-net.by
}

Поступила в редакцию 20.03.2018 г.

Показана возможность существования особого класса поверхностных электромагнитных волн, амплитуда которых неэкспоненциально убывает вглубь анизотропной металлодиэлектрической структуры, граничащей с изотропным диэлектриком. Получено дисперсионное уравнение, описывающее распространение таких особых поверхностных волн, а также найдены их коэффициенты затухания и вид электрического и магнитного полей в контактирующих средах. Получены выражения для продольной и поперечной компонент вектора Умова-Пойнтинга и объемной плотности электрической и магнитной энергии особых поверхностных волн. Проведены численные расчеты для слоистой металлодиэлектрической наноструктуры, описываемой одноосным тензором эффективной диэлектрической проницаемости.

DOI: $10.21883 /$ OS.2018.09.46553.80-18

\section{Введение}

Известно, что на границе раздела анизотропной и изотропной сред с положительно определенными диэлектрическими проницаемостями возможно существование поверхностных электромагнитных волн (ПЭВ). При этом их возбуждение возможно лишь вдоль некоторых направлений в плоскости раздела. Проблема возбуждения ПЭВ на границе оптически одноосного кристалла и изотропной среды рассматривалась в работах $[1,2]$. Особенности поверхностных волн, возникающих на границе одноосных кристаллов с различной ориентацией оптических осей, исследовались в [3-5].

Среди разнообразных ПЭВ особый интерес представляют поверхностные волны Дьяконова - локализованные моды, распространяющиеся в узкой области углов вдоль поверхности одноосного кристалла, содержащей его оптическую ось [2]. Экспериментально данные волны обнаружены в работе [6]. Волны Дьяконова вызывают значительный интерес в связи с возможностью их использования для передачи информации без потерь с субволновым разрешением. Следует отметить, что направлением распространения таких волн можно управлять, изменяя оптические свойства анизотропной среды, что достигается, например, при использовании вместо одноосных кристаллов наноструктур [7].

Рассмотренное к настоящему времени многообразие ПЭВ обладает тем общим свойством, что их амплитуда экспоненциально убывает при удалении от границы раз- дела. В настоящей работе рассмотрен новый тип ПЭВ, характеризующийся неэкспоненциальной зависимостью их электрического и магнитного полей от глубины проникновения в анизотропную среду.

\section{Условие существования поверхностных волн особого типа и их поляризационные и энергетические характеристики}

Пусть изотропная среда с диэлектрической проницаемостью $\varepsilon_{1}$ граничит с анизотропным оптически одноосным материалом, характеризуемым тензором диэлектрической проницаемости $\varepsilon=\operatorname{diag}\left\{\varepsilon_{o}, \varepsilon_{o}, \varepsilon_{e}\right\}=\varepsilon_{o}+\delta \mathbf{c} \cdot \mathbf{c}$, где $\delta=\varepsilon_{e}-\varepsilon_{o}, \mathbf{c}-$ единичный вектор вдоль оптической оси (точка между векторами обозначает их диадное произведение). В дальнейшем будем использовать систему координат, ось $Z$ которой направлена внутрь анизотропной среды, а ее начало совпадает с поверхностью раздела (рис. 1).

Рассмотрим возможность формирования на границе раздела указанных сред ПЭВ с неэкспоненциальной зависимостью амплитуд электрического (E) и магнитного $(\mathbf{H})$ полей от глубины проникновения в анизотропную среду.

Будем исходить из известного волнового уравнения для вектора $\mathbf{E}$, которое следует из уравнений Максвелла в случае гармонически зависящих от времени 


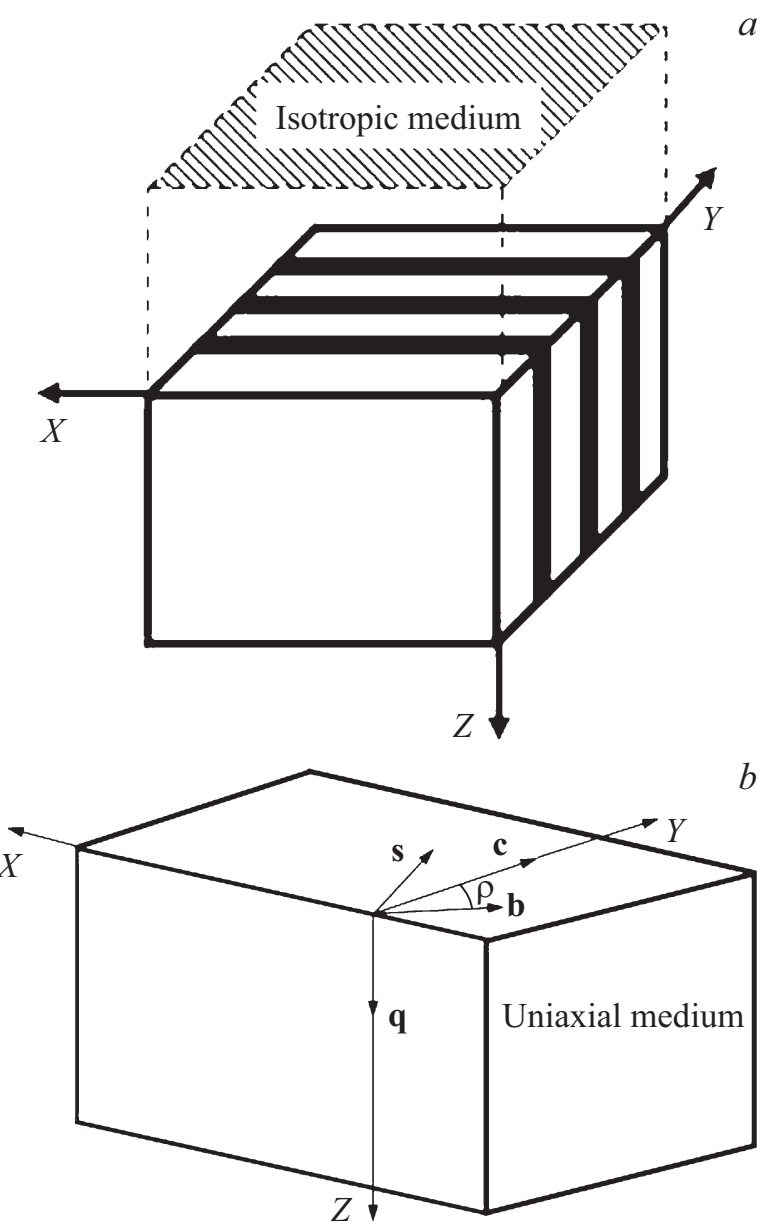

Рис. 1. Схема рассматриваемой структуры $(a)$ и ее представление в приближении эффективной среды $(b)$. c - единичный вектор вдоль оптической оси, $\mathbf{b}, \mathbf{q}, \mathbf{s}-$ тройка единичных векторов, где q - вектор, ортогональный границе раздела, $(\mathbf{c}, \mathbf{q})$ - главная плоскость анизотропной среды, $(\mathbf{b}, \mathbf{q})$ плоскость падения световой волны, повернутая относительно главной плоскости на угол $\rho$.

$(\sim \exp (-i \omega t))$ полей:

$$
\operatorname{grad} \operatorname{div} \mathbf{E}-\Delta \mathbf{E}-k^{2} \varepsilon \mathbf{E}=0,
$$

где $\varepsilon-$ тензор диэлектрической проницаемости, $k=\omega / c=2 \pi / \lambda$. Векторы напряженности электрического $\left(\mathbf{E}_{1}\right)$ и магнитного $\left(\mathbf{H}_{1}\right)$ полей волны в изотропной среде будем искать в виде

$$
\mathbf{E}_{1}=\left(A_{1} \mathbf{s}+\frac{1}{n_{1}} B_{1}\left[\mathbf{m}_{1} \mathbf{s}\right]\right) \exp \left(i k \mathbf{m}_{1} \mathbf{r}\right),
$$

$\mathbf{H}_{1}=\frac{1}{i k} \operatorname{rot} \mathbf{E}_{1}=\left[\mathbf{m}_{1} \mathbf{E}_{1}\right]=\left(A_{1}\left[\mathbf{m}_{1} \mathbf{s}\right]-n_{1} B_{1} \mathbf{s}\right) \exp \left(i k \mathbf{m}_{1} \mathbf{r}\right)$,

где вектор рефракции $\mathbf{m}_{1}=\xi \mathbf{b}-i \gamma_{1} \mathbf{q}\left(\mathbf{m}_{1}^{2}=\varepsilon_{1}=n_{1}^{2}=\right.$ $\left.=\xi^{2}-\gamma_{1}^{2}\right), \mathbf{b}, \mathbf{q}, \mathbf{s}-$ взаимно перпендикулярные орты, причем вектор $\mathbf{b}$ параллелен поверхности раздела сред, $\mathbf{q}$ - перпендикулярен к ней и направлен во вторую (анизотропную) среду (рис. 1$), A_{1}$ и $B_{1}$ - соответственно амплитуды перпендикулярной и параллельной плоскости падения составляющих волны.

В общем случае внутри анизотропной среды распространяются обыкновенная $(o)$ и необыкновенная $(e)$ волны, описываемые уравнением

$$
\mathbf{m}_{o}^{2}=\varepsilon_{o}, \quad \mathbf{m}_{e} \varepsilon \mathbf{m}_{e}=\varepsilon_{o} \varepsilon_{e}
$$

Здесь $\mathbf{m}_{o}=\xi \mathbf{b}+i \gamma_{o} \mathbf{q}-$ вектор рефракции волны $\left(\mathbf{m}_{o}^{2}=\varepsilon_{o}=\xi^{2}-\gamma_{o}^{2}\right)$. Однако в случае вырождения двулучепреломления, что имеет место при выполнении условия

$$
\left[\mathbf{m}_{o, e} \mathbf{c}\right]^{2}=0
$$

где $\mathbf{c}=(1 / \xi)\left(\varepsilon_{o}^{1 / 2} \mathbf{b}+\gamma_{o} \mathbf{s}\right)-$ единичный вектор вдоль оптической оси, как следует из теории дифференциальных уравнений, основы которой изложены в работе [8], а также из [9], решение волнового уравнения (1) для поля в одноосной среде представимо в виде

$$
\mathbf{E}_{2}=\left(\mathbf{f}_{1}+\sigma^{\prime} \mathbf{f}_{2}\right) \exp (i \sigma) .
$$

Здесь $\sigma=k \mathbf{m}_{o} \mathbf{r}, \sigma^{\prime}=k \mathbf{q r}, \mathbf{r}-$ радиус-вектор. Подставляя (5) в уравнение (1), получаем

$$
\boldsymbol{\theta} \mathbf{f}_{1}-i \boldsymbol{\beta} \mathbf{f}_{2}+\sigma^{\prime} \boldsymbol{\theta} \mathbf{f}_{2}=0
$$

Здесь $\boldsymbol{\theta}=\mathbf{m}_{o} \cdot \mathbf{m}_{o}-\mathbf{m}_{o}^{2}+\boldsymbol{\varepsilon}, \boldsymbol{\beta}=\mathbf{m}_{o} \cdot \mathbf{q}+\mathbf{q} \cdot \mathbf{m}_{o}-2 \mathbf{m}_{o} \mathbf{q}$. Уравнение (6) должно выполняться тождественно, т.е. при любых $\sigma^{\prime}$. Поэтому

$$
\boldsymbol{\theta} \mathbf{f}_{2}=\left(\mathbf{m}_{o} \cdot \mathbf{m}_{o}-\mathbf{m}_{o}^{2}+\boldsymbol{\varepsilon}_{o}+\delta \mathbf{c} \cdot \mathbf{c}\right) \mathbf{f}_{2}=0 .
$$

Так как для обыкновенной (а в рассматриваемом случае вырождения двулучепреломления и необыкновенной) волны $\mathbf{m}_{o}^{2}=\varepsilon_{o}$, то из $(7)$ следует $\left(\mathbf{m}_{o} \cdot \mathbf{m}_{o}+\delta \mathbf{c} \cdot \mathbf{c}\right) \mathbf{f}_{2}=0$. Отсюда видно, что вектор $\mathbf{f}_{2}$ коллинеарен вектору $\left[\mathbf{m}_{o} \mathbf{c}\right]$ и, следовательно,

$$
\mathbf{f}_{2}=t\left[\mathbf{m}_{o} \mathbf{c}\right] .
$$

Здесь $t-$ некоторый параметр. С учетом (8) уравнение (6) для $\mathbf{f}_{1}$ принимает вид

$$
\left(\mathbf{m}_{o} \cdot \mathbf{m}_{o}+\delta \mathbf{c} \cdot \mathbf{c}\right) \mathbf{f}_{1}-i\left(\mathbf{m}_{o} \cdot \mathbf{q}+\mathbf{q} \cdot \mathbf{m}_{o}-2 i \gamma_{o}\right) \mathbf{f}_{2}=0 .
$$

Для нахождения из (9) вектора $\mathbf{f}_{1}$ представим его в виде разложения по трем взаимно ортогональным ортам $\mathbf{b}, \mathbf{q}$ и $\mathbf{s :}$

$$
\mathbf{f}_{1}=t_{1} \mathbf{s}+t_{2} \mathbf{b}+t_{3} \mathbf{s}
$$

где $t_{1}, t_{2}, t_{3}$ - неизвестные величины. Подставляя $\mathbf{f}_{1}$ и $\mathbf{f}_{2}$ в исходное уравнение (9) и приравнивая нулю коэффициенты при $\mathbf{b}, \mathbf{q}$ и $\mathbf{s}$, можно выразить $t_{2}, t_{3}$ через $t_{1}, t$ и, следовательно, определить $\mathbf{f}_{1}$. В результате получаем

$$
\mathbf{f}_{1}=\frac{i}{\delta \sqrt{\varepsilon_{o}}}\left\{\frac{\xi \delta}{\gamma}\left[\mathbf{m}_{o} \mathbf{c}\right] t_{1}+i \sqrt{\varepsilon_{o}}\left(2\left[\mathbf{m}_{o} \mathbf{s}\right]-\delta \mathbf{q}\right) t\right\} .
$$


При этом с учетом (8), (11) выражения для векторов электрической $\mathbf{E}_{2}$ и магнитной $\mathbf{H}_{2}$ напряженностей принимают следующий вид [9]:

$$
\begin{aligned}
\mathbf{E}_{2}= & \left\{C_{1}\left[\mathbf{m}_{o} \mathbf{c}\right]+C_{2}\left(\mathbf{d} \varsigma^{\prime}\left[\mathbf{m}_{o} \mathbf{c}\right]\right)\right\} \exp \left(i k \mathbf{m}_{o} \mathbf{r}\right), \\
\mathbf{H}_{2}= & \frac{1}{i k} \operatorname{rot} \mathbf{E}_{2}=\left\{C_{1}\left[\mathbf{m}_{o}\left[\mathbf{m}_{o} \mathbf{c}\right]\right]\right. \\
& \left.+C_{2}\left(\mathbf{d}_{1}+\delta \varsigma^{\prime}\left[\mathbf{m}_{o}\left[\mathbf{m}_{o} \mathbf{c}\right]\right]\right)\right\} \exp \left(i k \mathbf{m}_{o} \mathbf{r}\right)
\end{aligned}
$$

Здесь приняты обозначения $\mathbf{d}=\delta \mathbf{q}-2 \xi\left[\mathbf{m}_{o} \mathbf{s}\right], \mathbf{d}_{1}=$ $=\left[\mathbf{m}_{o} \mathbf{d}\right]-i \delta\left[\mathbf{q}\left[\mathbf{m}_{o} \mathbf{c}\right]\right]=\left[\mathbf{m}_{o} \mathbf{d}\right]-\delta \gamma \mathbf{c}$, а вместо сомножителей при векторных величинах в выражении для $\mathbf{f}_{1}$ введены амплитуды волны $C_{1}, C_{2}$, которые определяются из граничных условий.

Подставляя (2) и (12) в стандартные граничные условия, получаем (при $z=0$ ) два векторных уравнения, из которых следует система алгебраических уравнений для нахождения $A_{1}\left(B_{1}\right)$ и $C_{1}\left(C_{2}\right)$ :

$$
\begin{gathered}
\xi A_{1}+i \gamma_{o} \sqrt{\varepsilon_{o}} C_{1}=0, \\
i \gamma_{1} \xi A_{1}+\gamma_{o}^{2} \sqrt{\varepsilon_{o}} C_{1}-\delta \gamma_{o} \sqrt{\varepsilon_{o}} C_{2}=0, \\
\xi \gamma_{1} B_{1}+n_{1} \gamma_{o}^{2} C_{1}-2 n_{1} \xi^{2} \gamma_{o} C_{2}=0, \\
\xi n_{1} B_{1}-\varepsilon_{o} \gamma_{o} C_{1}+\varepsilon_{o}\left(\delta+2 \xi^{2}\right) C_{2}=0 .
\end{gathered}
$$

Здесь учтено, что в этом случае qс $=0$. Исключая из первых двух уравнений $A_{1}$, а из последних двух $B_{1}$, получаем для $C_{1}$ и $C_{2}$ соотношения

$$
C_{2}=\frac{\gamma_{o}+\gamma_{1}}{\delta} C_{1}, \quad C_{2}=\frac{\gamma_{o}\left(\varepsilon_{1} \gamma_{o}+\varepsilon_{o} \gamma_{1}\right)}{\varepsilon_{o} \gamma_{1} \delta+2 \xi^{2}\left(\varepsilon_{1} \gamma_{o}+\varepsilon_{o} \gamma_{1}\right)} C_{1},
$$

где $C_{1}=i \xi A_{1} /\left(\gamma_{o} \sqrt{\varepsilon_{o}}\right)$. Кроме того, из системы (13) следует выражение, связывающее амплитуды $A_{1}$ и $B_{1}$ :

$$
\begin{gathered}
B_{1} / A_{1}=\kappa=i M, \\
M=\left[2 \xi^{2}\left(\gamma_{o}+\gamma_{1}\right)-\delta \gamma_{o}\right] \sqrt{\varepsilon_{1} / \varepsilon_{o}} /\left(\delta \gamma_{1}\right) .
\end{gathered}
$$

Как видно, волна в первой среде является эллиптически поляризованной вследствие комплексности параметра $\kappa$.

Сравнивая оба выражения (14) для $C_{2}$, приходим к соотношению

$$
\delta\left(\varepsilon_{1} \gamma_{o}^{2}-\varepsilon_{o} \gamma_{1}^{2}\right)=2 \xi^{2}\left(\gamma_{o}+\gamma_{1}\right)\left(\varepsilon_{1} \gamma_{o}+\varepsilon_{o} \gamma_{1}\right)
$$

которое можно преобразовать к более простому виду с учетом выражений для $\gamma_{o}^{2}$ и $\gamma_{1}^{2}$ :

$$
\delta=\varepsilon_{e}-\varepsilon_{o}=2\left(\varepsilon_{1} \gamma_{o}+\varepsilon_{o} \gamma_{1}\right) /\left(\gamma_{o}-\gamma_{1}\right) .
$$

Выполнение условий (17) и $\gamma_{o}>\gamma_{1}>0$ необходимо для локализации вблизи поверхности раздела изотропного диэлектрика и анизотропной структуры поверхностных электромагнитных волн особого типа, подобных неоднородной волне, возникающей при полном отражении света от оптически одноосного кристалла. Это выполняется при $\delta>2 \varepsilon_{1}>2 \varepsilon_{0}>0$, т. е. при достаточно больших значениях $\delta$, что практически невозможно для всех традиционно используемых в кристаллооптике анизотропных материалов, для которых $\delta=\varepsilon_{e}-\varepsilon_{0}$, как правило, весьма мало.

При заданных параметрах граничащих сред $\varepsilon_{o}, \varepsilon_{e}$ и $\varepsilon_{1}$ из (17) нетрудно получить выражение для постоянной распространения $\xi$ поверхностной волны. Оно имеет следующий вид:

$$
\xi^{2}=\frac{\left(\varepsilon_{1}-\varepsilon_{o}\right) \delta^{2}+8 \varepsilon_{o} \varepsilon_{1} \delta-4 \varepsilon_{0} \varepsilon_{1}\left(\varepsilon_{1}-\varepsilon_{o}\right)}{4\left(\varepsilon_{1}-\varepsilon_{o}\right)\left[\delta-\left(\varepsilon_{1}-\varepsilon_{o}\right)\right]} .
$$

Анализ выражения (18) показывает, что $\xi^{2}>\varepsilon_{1}$ при $\delta>2 \varepsilon_{1}>2 \varepsilon_{0}>0$. Из системы (13) следует также, что она имеет ненулевые решения только при отличном от нуля значении амплитуды $C_{1}$.

Таким образом, соотношение (18) является по сути дисперсионным уравнением для определения скорости $V(\omega)=c / \xi(\omega)$ распространения поверхностной волны, при этом $\xi$ имеет смысл эффективного показателя преломления данной ПЭВ. Согласно (2) и (12), рассматриваемая ПЭВ является особой волной, поскольку характеризуется неэкспоненциальным затуханием амплитуды при удалении от границы одноосного кристалла. Эллиптичность (отношение большой $(a)$ и малой $(b)$ полуосей эллипса) поверхностной волны, а также угол наклона $\chi$ большой оси эллипса к плоскости падения можно рассчитать с помощью соотношений [10]

$$
\left(\frac{b}{a}\right)^{2}=\frac{|\mathbf{E}|^{2}-\left|\mathbf{E}^{2}\right|}{|\mathbf{E}|^{2}+\left|\mathbf{E}^{2}\right|}=M^{2}, \quad \operatorname{tg} 2 \chi=\frac{\kappa+\kappa^{*}}{1-|\kappa|^{2}},
$$

откуда следует

$$
\begin{gathered}
\left(\frac{b}{a}\right)^{2}=\left[2 \xi^{2}\left(\gamma_{o}+\gamma_{1}\right)-\delta \gamma_{o}\right]^{2} \varepsilon_{1} /\left(\varepsilon_{o} \delta^{2} \gamma_{1}^{2}\right), \\
\operatorname{tg} 2 \chi=0, \quad \chi=0 .
\end{gathered}
$$

Рассмотрим далее вопрос о скорости распространения рассматриваемой поверхностной волны. Для этого найдем сначала средние по времени локальные значения распространяющегося вдоль поверхности раздела сред потока $\overline{\mathbf{S}}$ и плотности энергии $\bar{W}=\bar{W}_{e}+\bar{W}_{m}$, где $\bar{W}_{e}$ и $\bar{W}_{m}$ - соответственно плотности электрической и магнитной энергии (черта сверху означает усредненные по периоду колебаний величины). При этом

$$
\begin{gathered}
\bar{W}_{e}=\frac{1}{16 \pi} \operatorname{Re}\left(\mathbf{E D}^{*}\right), \quad \bar{W}_{m}=\frac{1}{16 \pi}|\mathbf{H}|^{2}, \\
\overline{\mathbf{S}}=\frac{c}{8 \pi} \operatorname{Re}[\mathbf{E H}] .
\end{gathered}
$$


Раскрывая эти выражения с учетом (12), получим

$$
\begin{aligned}
& \bar{W}_{e}=\frac{\varepsilon_{o}}{16 \pi}\left\{2 \gamma_{o}^{2}\left|C_{1}\right|^{2}+\left[\delta^{2}+4\left(\delta+\xi^{2}\right)\left(\xi^{2}+\gamma_{o}^{2}\right)\right.\right. \\
& \left.-2 \delta\left(\delta+2 \xi^{2}+2 \gamma_{o}^{2}\right) \gamma_{o} \varsigma^{\prime}+2 \delta^{2}\left(\gamma_{o} \varsigma^{\prime}\right)^{2}\right]\left|C_{2}\right|^{2} \\
& \left.-2 \gamma_{0}\left(\delta+2 \xi^{2}+2 \gamma_{o}^{2}-2 \delta \gamma_{o} \varsigma^{\prime}\right) C_{1} C_{2}^{*}\right\} \exp \left(-2 k \gamma_{o} z\right) \\
& \bar{W}_{m}=\frac{\varepsilon_{o}}{16 \pi}\left\{2 \gamma_{o}^{2}\left|C_{1}\right|^{2}+\left[\delta^{2}+4 \varepsilon_{o}\left(\delta+\xi^{2}\right)\right.\right. \\
& \left.-2 \delta\left(\varepsilon_{o}+\varepsilon_{e}\right) \gamma_{o} \varsigma^{\prime}+2 \delta^{2}\left(\gamma_{0} \varsigma^{\prime}\right)^{2}\right]\left|C_{2}\right|^{2} \\
& \left.-2 \gamma_{0}\left(\varepsilon_{o}+\varepsilon_{e}-2 \delta \gamma_{o} \varsigma^{\prime}\right) C_{1} C_{2}^{*}\right\} \exp \left(-2 k \gamma_{o} z\right)
\end{aligned}
$$

Тогда для суммарной локальной плотности энергии имеем

$$
\begin{aligned}
\bar{W}= & \frac{\varepsilon_{o}}{8 \pi}\left\{2 \gamma_{o}^{2}\left|C_{1}\right|^{2}+\left[\left(\delta+2 \xi^{2}\right)^{2}\right.\right. \\
& \left.-2 \delta\left(\delta+2 \xi^{2}\right) \gamma_{o} \varsigma^{\prime}+2 \delta^{2}\left(\gamma_{o} \varsigma^{\prime}\right)^{2}\right]\left|C_{2}\right|^{2} \\
& \left.-2 \gamma_{0}\left(\delta+2 \xi^{2}-2 \delta \gamma_{o} \varsigma^{\prime}\right) C_{1} C_{2}^{*}\right\} \exp \left(-2 k \gamma_{o} z\right) .
\end{aligned}
$$

Для переносимого поверхностной волной потока энергии $\overline{\mathbf{S}}_{1}$ в направлении вектора $\mathbf{b}$ в этом случае получаем следующее выражение:

$$
\begin{aligned}
\overline{\mathbf{S}}_{1} & =\frac{c \varepsilon_{o}}{8 \pi \xi}\left\{2 \gamma_{o}^{2}\left|C_{1}\right|^{2}+\left[\left(\delta+2 \xi^{2}\right)^{2}\right.\right. \\
& \left.-2 \delta\left(\delta+2 \xi^{2}\right) \gamma_{o} \varsigma^{\prime}+2 \delta^{2}\left(\gamma_{0} \varsigma^{\prime}\right)^{2}\right]\left|C_{2}\right|^{2} \\
& \left.-2 \gamma_{0}\left(\delta+2 \xi^{2}-2 \delta \gamma_{o} \varsigma^{\prime}\right) C_{1} C_{2}^{*}\right\} \exp \left(-2 k \gamma_{o} z\right) \mathbf{b} .
\end{aligned}
$$

Из сравнения (24) и (23) следует, что $\overline{\mathbf{S}}_{1}=V \bar{W} \mathbf{b}$, где $V=c / \xi-$ скорость распространения продольного потока, переносимого поверхностной волной.

Отметим, что, наряду с продольным потоком $\overline{\mathbf{S}}_{1}$ в этом случае существует также поток $\overline{\mathbf{S}}_{2}$, перпендикулярный плоскости падения. Расчет дает для него выражение

$$
\begin{aligned}
\overline{\mathbf{S}}_{2} & =\frac{c \gamma_{o} \sqrt{\varepsilon_{o}}}{8 \pi \xi}\left\{2 \gamma_{o}^{2}\left|C_{1}\right|^{2}+\delta\left[\delta+2 \xi^{2}\right.\right. \\
& \left.-2\left(\delta+2 \xi^{2}\right) \gamma_{o} \varsigma^{\prime}+2 \delta\left(\gamma_{0} \varsigma^{\prime}\right)^{2}\right]\left|C_{2}\right|^{2} \\
& \left.-2 \gamma_{o}\left[2 \xi^{2}+\delta\left(1-2 \gamma_{o} \varsigma^{\prime}\right)\right] C_{1} C_{2}^{*}\right\} \exp \left(-2 k \gamma_{o} z\right) \mathbf{s} .
\end{aligned}
$$

Наличие так называемой боковой составляющей потока поверхностной волны приводит к эффекту бокового смещения светового луча, т.е. выходу его из плоскости падения. Этот эффект впервые был теоретически предсказан Ф.И. Федоровым при исследовании явления полного отражения света на границе раздела двух изотропных сред [10].

Что касается нормальной составляющей потока $\overline{\mathbf{S}}_{n}$, ортогональной границе раздела двух сред ( $\mid \mathbf{q})$, то, как и в случае полного отражения света, она отсутствует $\left(\overline{\mathbf{S}}_{n} \mathbf{q}=0\right)$.

Рассмотрим теперь поток $\overline{\mathbf{S}}$ и плотность энергии $\bar{W}=\bar{W}_{e}+\bar{W}_{m}$ в первой (изотропной) среде. Подставляя в (22), (23) выражения $\mathbf{E}_{1}$ и $\mathbf{H}_{1}$ из (1), находим

$$
\begin{gathered}
\bar{W}_{e}^{(1)}=\frac{1}{16 \pi}\left\{\varepsilon_{1}+\left(\xi^{2}+\gamma_{1}^{2}\right)|\kappa|^{2}\right\}\left|A_{1}\right|^{2} \exp \left(2 k \gamma_{1} z\right) \\
\bar{W}_{m}^{(1)}=\frac{1}{16 \pi}\left\{2 \xi^{2}-\varepsilon_{1}+\left(\xi^{2}-\gamma_{1}^{2}\right)|\kappa|^{2}\right\}\left|A_{1}\right|^{2} \exp \left(2 k \gamma_{1} z\right) \\
\bar{W}^{(1)}=\frac{\xi^{2}}{8 \pi}\left\{1+|\kappa|^{2}\right\}\left|A_{1}\right|^{2} \exp \left(2 k \gamma_{1} z\right)
\end{gathered}
$$

Продольная компонента вектора Умова-Пойнтинга определяется при этом выражением

$$
\overline{\mathbf{S}}_{1}^{(1)}=\frac{c \xi}{8 \pi}\left\{1+|\kappa|^{2}\right\}\left|A_{1}\right|^{2} \exp \left(2 k \gamma_{1} z\right) \mathbf{b}=V \bar{W}^{(1)} \mathbf{b} .
$$

Соответствующие средние интегральные плотности энергии $W$ и потока энергии $\mathbf{S}$ в изотропной среде

$$
\begin{gathered}
W^{(1)}=\frac{\xi^{2}}{8 \pi k}\left\{1+|\kappa|^{2}\right\}\left|A_{1}\right|^{2} \int_{-\infty}^{0} \exp \left(2 \gamma_{1} k z\right) d z \\
=\frac{\xi^{2}}{16 \pi \gamma_{1} k}\left\{1+|\kappa|^{2}\right\}\left|A_{1}\right|^{2}, \\
\mathbf{S}_{1}^{(1)}=\frac{c \xi}{8 \pi}\left\{1+|\kappa|^{2}\right\}\left|A_{1}\right|^{2} \int_{-\infty}^{0} \exp \left(2 \gamma_{1} k z\right) d z \mathbf{b} \\
=\frac{c \xi}{16 \pi \gamma_{1}}\left\{1+|\kappa|^{2}\right\}\left|A_{1}\right|^{2} \mathbf{b} .
\end{gathered}
$$

Для анизотропной среды, аналогично интегрируя выражения (21), (22) с учетом (15), получим

$$
\begin{gathered}
W^{(2)}=\frac{\xi^{2}}{16 \gamma_{o}^{3} \delta^{2} \pi k}\left\{2 \gamma_{o}^{2} \delta^{2}+\left(\gamma_{o}+\gamma_{1}\right)\right. \\
\left.\times\left[\left(\gamma_{o}+\gamma_{1}\right)\left(\delta^{2}+2 \delta \xi^{2}+4 \xi^{4}\right)+4 \gamma_{o} \delta\left(\delta+\xi^{2}\right)\right]\right\}\left|A_{1}\right|^{2} \\
\mathbf{S}_{1}^{(2)}=\frac{c}{\xi} W^{(2)} \mathbf{b} .
\end{gathered}
$$

Полные плотность энергии поверхностной волны $W=W^{(1)}+W^{(2)}$ и продольный поток $\mathbf{S}_{1}=\mathbf{S}_{1}^{(1)}+\mathbf{S}_{1}^{(2)}$ определяются следующими выражениями:

$$
\begin{gathered}
W=\frac{\xi^{2}\left|A_{1}\right|^{2}}{16 \pi k}\left\{\frac{1}{\gamma_{1}}\left(1+|\kappa|^{2}\right)+\frac{1}{\gamma_{o}^{3} \delta^{2}}\left[2 \gamma_{o}^{2} \delta^{2}+\left(\gamma_{o}+\gamma_{1}\right)\right.\right. \\
\left.\left.\times\left[\left(\gamma_{o}+\gamma_{1}\right)\left(\delta^{2}+2 \delta \xi^{2}+4 \xi^{4}\right)+4 \gamma_{o} \delta\left(\delta+\xi^{2}\right)\right]\right]\right\} \\
\mathbf{S}_{1}=\frac{c}{\xi}\left(W^{(1)}+W^{(2)}\right) \mathbf{b} .
\end{gathered}
$$




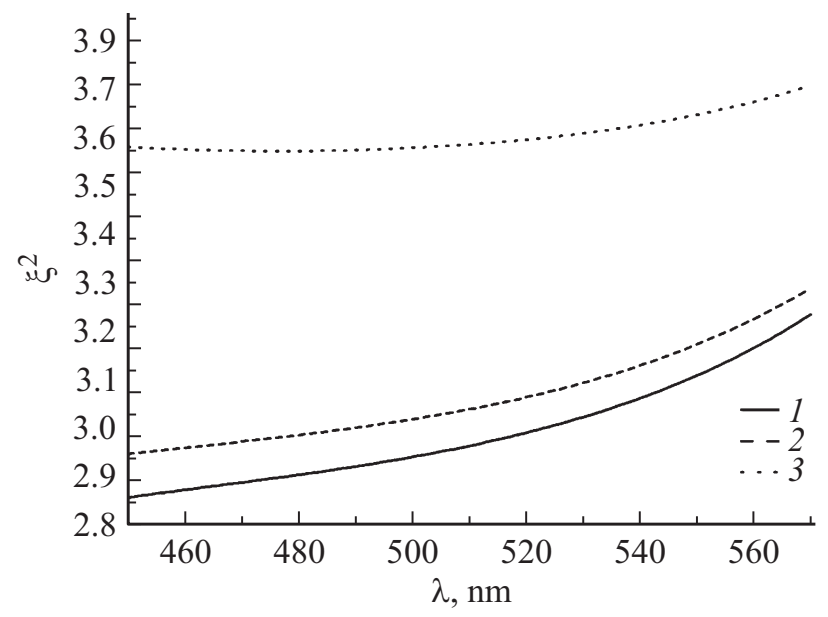

Pис. 2. Спектральная зависимость квадрата константы распространения поверхностной волны особого типа, генерируемой на границе раздела металлодиэлектрической наноструктуры $\mathrm{TiO}_{2} / \mathrm{Ag}\left(d_{m}=20 \mathrm{~nm}, f=0.3, \varepsilon_{\infty}=5, \varepsilon_{d}=5.913\right)$ и изотропного диэлектрика: 1 - оптическое стекло ВАF $10, \varepsilon_{1}=2.789$, 2 - оптическое стекло SF $5, \varepsilon_{1}=2.8761,3$ - оптическое стекло LASF 9, $\varepsilon_{1}=3.5329$.

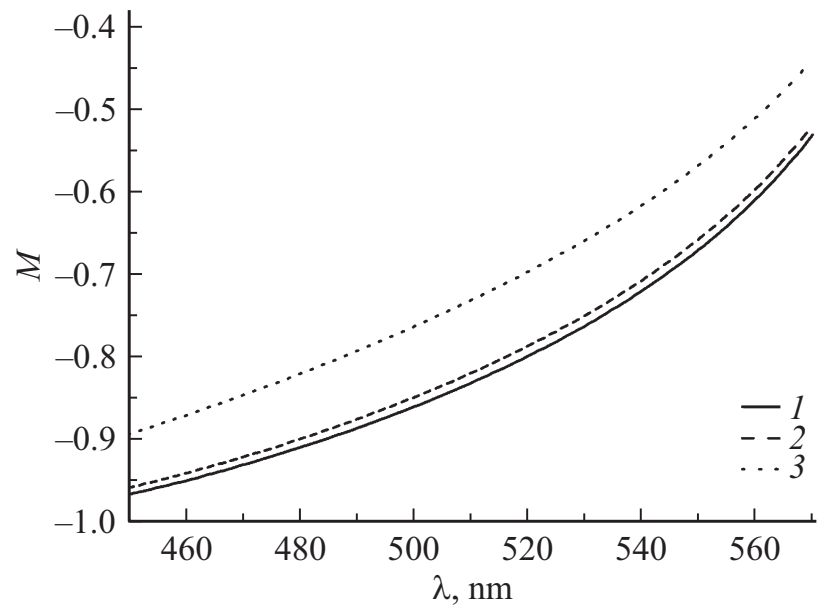

Pис. 3. Спектральная зависимость эллиптичности поверхностной волны особого типа, генерируемой на границе раздела металлодиэлектрической наноструктуры $\mathrm{TiO}_{2} / \mathrm{Ag}\left(d_{m}=20 \mathrm{~nm}\right.$, $\left.f=0.3, \varepsilon_{\infty}=5, \varepsilon_{d}=5.913\right)$ и изотропного диэлектрика: $1-$ оптическое стекло BAF $10, \varepsilon_{1}=2.789,2$ - оптическое стекло SF $5, \varepsilon_{1}=2.8761,3-$ оптическое стекло LASF $9, \varepsilon_{1}=3.5329$.

\section{Результаты расчетов для поверхностной волны особого вида}

Рассмотрим слоисто-периодическую анизотропную наноструктуру, образованную чередующимися слоями металла и диэлектрика. В приближении эффективной среды, когда толщина каждого слоя достаточно мала, т. е. $\left|k_{d} d_{d}\right| \ll 1,\left|k_{m} d_{m}\right| \ll 1$, где $k_{d}, k_{m}-$ соответственно волновые числа диэлектрического и металлического слоев, многослойная структура может рассматриваться как эффективная оптически одноосная среда, тензор диэлек- трической проницаемости которой имеет собственные значения, определяемые выражением

$$
\varepsilon_{o}=(1-f) \varepsilon_{d}+f \varepsilon_{m}, \quad \varepsilon_{e}=\left[\frac{1-f}{\varepsilon_{d}}+\frac{f}{\varepsilon_{m}}\right]^{-1} .
$$

Здесь $f=d_{m} /\left(d_{m}+d_{d}\right)$ - фактор заполнения (объемная доля, занимаемая в структуре металлом), $\varepsilon_{d}, \varepsilon_{m}-$ соответственно проницаемости диэлектрического и металлического слоев. При этом величина $\varepsilon_{m}$ описывается формулой Друде:

$$
\begin{aligned}
\varepsilon_{m}(\omega) & =\varepsilon_{\infty}-\omega_{p}^{2} /\left(\omega^{2}+i \omega \Gamma\right) \\
& =\varepsilon_{\infty}-\omega_{p}^{2} /\left(\omega^{2}+\Gamma^{2}\right)+i \omega_{p}^{2} \Gamma /\left[\omega\left(\omega^{2}+\Gamma^{2}\right)\right]
\end{aligned}
$$

где $\omega_{p}-$ плазменная частота, $\varepsilon_{\infty}-$ постоянная, описывающая вклад межзонных переходов, $\Gamma=V_{F} / l-$ постоянная затухания, $V_{F}-$ скорость Ферми, $l-$ среднее
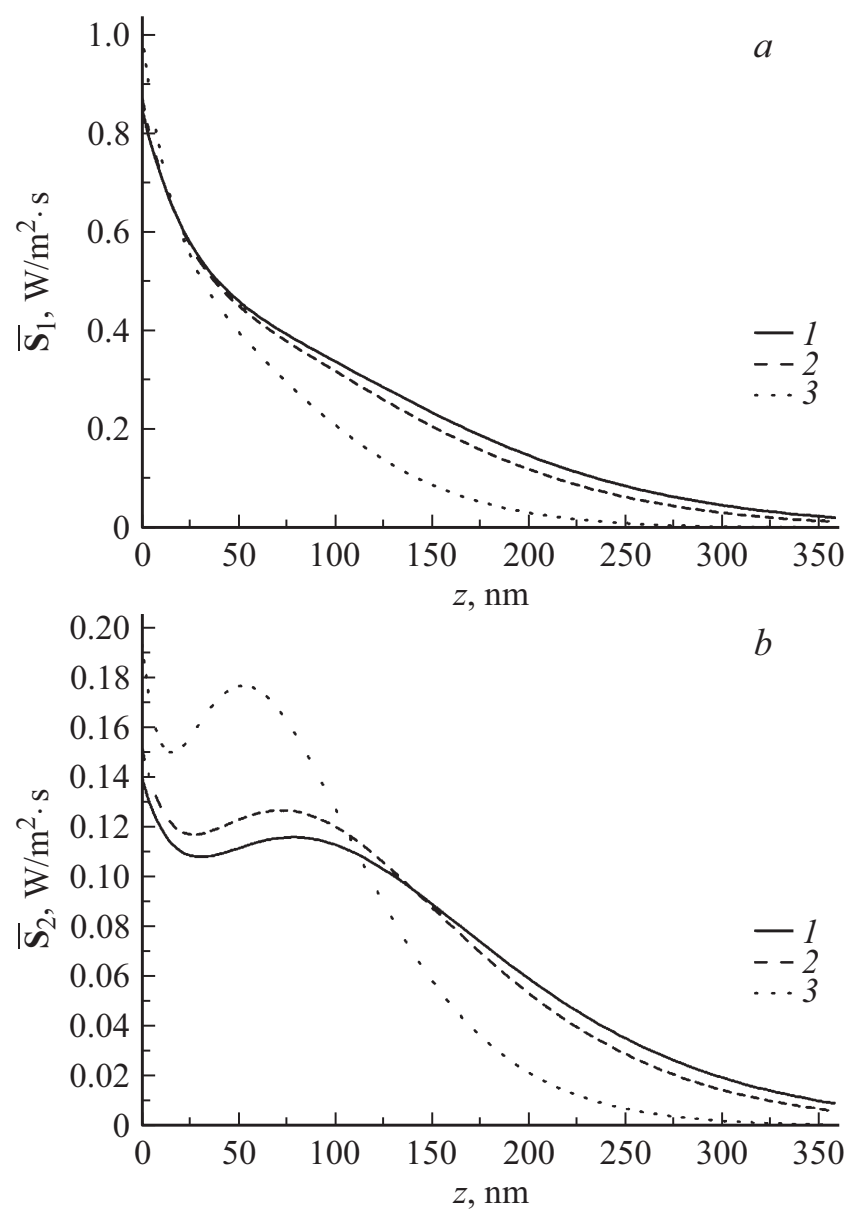

Рис. 4. Зависимость от глубины проникновения внутрь анизотропной среды продольного $(a)$ и поперечного $(b)$ энергетического потока поверхностной волны особого типа, генерируемой на границе раздела металлодиэлектрической наноструктуры $\mathrm{TiO}_{2} / \mathrm{Ag}\left(d_{m}=20 \mathrm{~nm}, f=0.3, \lambda=450 \mathrm{~nm}, \varepsilon_{\infty}=5\right.$, $\left.\varepsilon_{d}=5.913, \varepsilon_{e}=15.021, \varepsilon_{o}=2.403\right)$ и изотропного диэлектрика: 1 - оптическое стекло ВАF $10, \varepsilon_{1}=2.789,2$ - оптическое стекло SF $5, \varepsilon_{1}=2.8761,3$ - оптическое стекло LASF 9, $\varepsilon_{1}=3.5329$. 

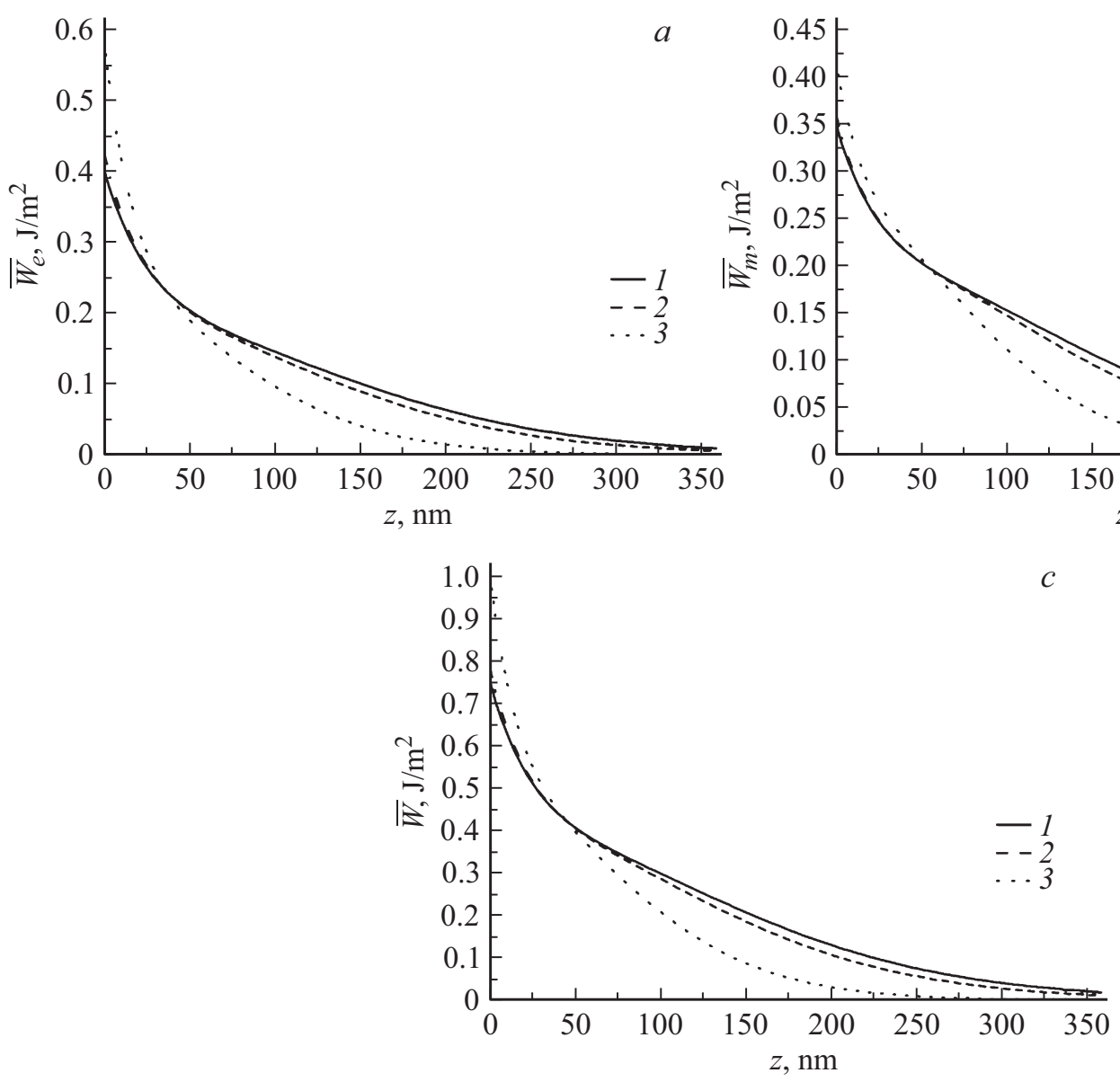

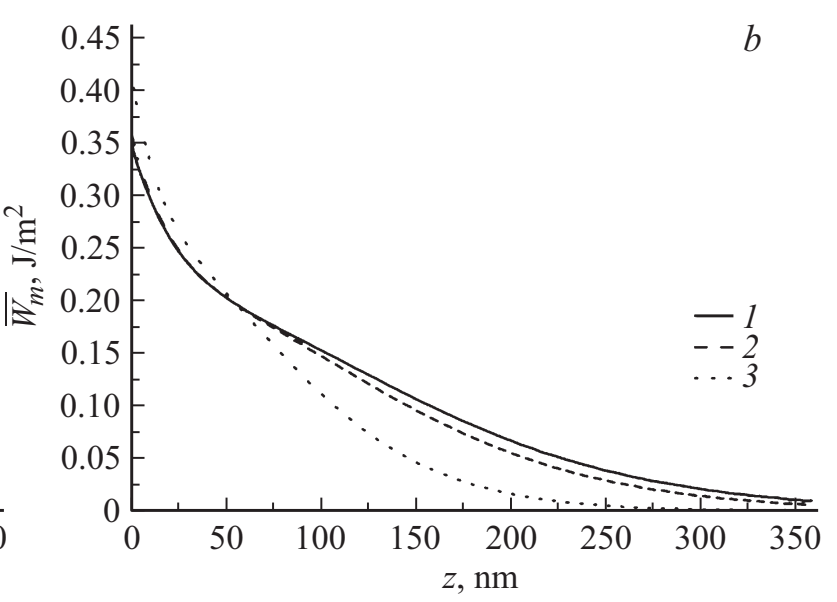

Рис. 5. Зависимость от глубины проникновения внутрь анизотропной среды плотности электрической $(a)$, магнитной $(b)$ и полной $(c)$ энергии поверхностной волны особого типа, генерируемой на границе раздела металлодиэлектрической наноструктуры $\mathrm{TiO}_{2} / \mathrm{Ag}\left(d_{m}=20 \mathrm{~nm}, f=0.3, \lambda=450 \mathrm{~nm}, \varepsilon_{\infty}=5, \varepsilon_{d}=5.913, \varepsilon_{e}=15.021, \varepsilon_{o}=2.403\right)$ и изотропного диэлектрика: 1 оптическое стекло BAF $10, \varepsilon_{1}=2.789,2$ - оптическое стекло SF $5, \varepsilon_{1}=2.8761,3$ - оптическое стекло LASF 9, $\varepsilon_{1}=3.5329$.

значение свободного пути электрона в объемном металле. Для серебра, например, $\varepsilon_{\infty}=5, \omega_{p}=14 \cdot 10^{15} \mathrm{~s}^{-1}$, $\Gamma=32 \cdot 10^{12} \mathrm{~s}^{-1}, V_{F}=1.4 \cdot 10^{6} \mathrm{~ms}^{-1}$ [11]. Расчет, проведенный для слоисто-периодической среды $\mathrm{TiO}_{2} / \mathrm{Ag}$ $\left(d_{m}=20 \mathrm{~nm}, f=0.3, \varepsilon_{d}=5.913\right)$, показывает, что для данной структуры в диапазоне длин волн $450 \mathrm{~nm} \leq \lambda \leq 560 \mathrm{~nm}$ величины $\varepsilon_{o}, \varepsilon_{e}$ положительны, причем $\varepsilon_{e} \gg \varepsilon_{o}$ и поглощением материала можно пренебречь. В этом спектральном диапазоне $\varepsilon_{e}>2 \varepsilon_{1}+\varepsilon_{o}>3 \varepsilon_{o}>0$, следовательно, оказывается возможным возбуждение поверхностной волны. При этом с увеличением диэлектрической проницаемости граничащего с наноструктурой диэлектрика константа ее распространения возрастает (рис. 2), а эллиптичность убывает (рис. 3).

Расчет плотностей и потоков энергии проведен для трех граничащих со структурой изотропных диэлектриков: 1 - оптическое стекло BAF $10, \varepsilon_{1}=2.789,2-$ оптическое стекло SF $5, \varepsilon_{1}=2.8761,3-$ оптическое стекло LASF 9, $\varepsilon_{1}=3.5329$. Во всех случаях значение амплитуды волны $A_{1}$ в диэлектрике считалось одинако- вым, таким, что при $z=0$ значения $\bar{W}$ и $\overline{\mathbf{S}}_{1}$ в случае 3 равны соответственно $1 \mathrm{~J} / \mathrm{m}^{3}$ и $1 \mathrm{~W} / \mathrm{m}^{2} \mathrm{~s}$.

Следует отметить, что с увеличением длины световой волны имеет место возрастание постоянной распространения поверхностной волны (рис. 2). При этом с увеличением диэлектрической проницаемости изотропной среды увеличивается спектральный диапазон, где данная зависимость весьма слабая.

На рис. 4 приведены зависимости продольного и поперечного энергетических потоков поверхностной волны от глубины проникновения в металлодиэлектрическую структуру. Видно, что в некотором диапазоне проницаемостей изотропной среды, граничащей с металлодиэлектрической структурой, наблюдается неэкспоненциальное уменьшение как продольного, так и поперечного потоков энергии. При этом величина поперечного потока $\mathbf{S}_{2}$ достигает максимума не на поверхности раздела, а на некотором расстоянии $Z_{\max }$ вблизи нее. Отметим, что $Z_{\max }$ увеличивается при возрастании (рис. $4, b$ ).

На рис. 5 представлены зависимости объемной плотности электрической, магнитной и полной энергии по- 
верхностной волны от глубины ее проникновения внутрь анизотропной среды.

Из рис. 5 видно, что существуют области значений диэлектрических проницаемостей граничащего с металлодиэлектрической структурой диэлектрика, для которых ПЭВ неэкспоненциально затухает.

\section{Заключение}

Таким образом, в данной работе показана возможность возбуждения на границе изотропной среды и слоистой металлодиэлектрической наноструктуры, характеризуемой одноосным эффективным тензором диэлектрической проницаемости с положительными собственными значениями, поверхностной волны особого типа, амплитуда которой убывает внутри анизотропной структуры при удалении от ее поверхности по сложному неэкспоненциальному закону. Найдены условия существования такой волны.

Получены выражения для продольной и поперечной компонент вектора Умова-Пойнтинга и объемной плотности электрической и магнитной энергии. Установлено наличие компоненты потока энергии, перпендикулярной направлению распространения ПЭВ вдоль границы раздела сред, вследствие чего имеет место эффект бокового смещения энергии.

С использованием полученных выражений осуществлено численное моделирование характеристик особых поверхностных волн, формируемых на границе диэлектрика и слоисто-периодической металлодиэлектрической наноструктуры $\mathrm{TiO}_{2} / \mathrm{Ag}$. Установлено, что формируемая поверхностная волна характеризуется неэкспоненциальным уменьшением продольного энергетического потока, а поперечный (смещающий) поток энергии появлением четко выраженного максимума вблизи поверхности наноструктуры. При увеличении проницаемости граничащего изотропного диэлектрика этот максимум увеличивается и смещается к границе раздела металлодиэлектрической наноструктуры и изотропной среды.

Полученные результаты имеют перспективы применения при создании высокочувствительных сенсоров, основанных на использовании особых поверхностных волн. Они могут быть использованы также при разработке новых методов зондирования приповерхностных дефектов различных материалов.

\section{Список литературы}

[1] Марчевский Ф.Н., Стрижевский В.Л., Стрижевский С.В. // ФТТ. 1984. Т. 26. С. 1501.

[2] Дьяконов М.И. // ЖЭТФ. 1988. Т. 94. № 4. С. 119.

[3] Аверкиев Н.С., Дьяконов М.И. // Опт. и спектр. 1990. Т. 68. C. 1118.

[4] Даринский А.Н. // Кристаллография. 2001. Т. 46. С. 916.
[5] Фурс А.Н., Галынский В.М., Барковский Л.М. // Опт. и спектр. 2005. T. 98. С. 500; Furs A.N., Galynsky V.M., Barkovsky L.M. // Opt. Spectrosc. 2005. V. 98. P. 454. doi 10.1134/1.1890527

[6] Takayama O., Crasovan L., Artigas D., Torner L. // Phys. Rev. Lett. 2009. V. 102. P. 043903. doi 10.1103/PhysRevLett.102.043903

[7] Takayama O., Artigas D., Torner L. // Nature Nanotechnology. 2014. V. 9. P. 419. doi 10.1038/nnano.2014.90

[8] Kelley W.G., Peterson A.C. The Theory of Differential Equations: Classical and Qualitative. New York: SpringerVerlag, 2010. doi 10.1007/978-1-4419-5783-2.

[9] Kurilkina S.N., Petrov N.S., Zimin A.B., Belyi V.N. // J. Opt. 2017. V. 19. P. 125102. doi 10.1088/2040-8986/aa945c

[10] Федоров Ф.И. Оптика анизотропных сред. Мн.: АН БССР, 1958. $381 \mathrm{c}$.

[11] Cai W., Shalaev V. Optical Metamaterials: Fundamentals and Applications. Springer, 2010. doi 10.1007/978-1-4419-1151-3 